\title{
Risk Curve and Bifuzzy Portfolio Selection
}

\author{
Limei Yan \\ Department of Mathematics, Dezhou University \\ Dezhou 253023, China \\ E-mail: yanlimei9898@163.com
}

\begin{abstract}
In order to solve the portfolio problem when security returns are bifuzzy variables, firstly we propose a new definition of risk, then one type of portfolio selection based on expected value and risk is provided according to bifuzzy theory. Furthermore, a hybrid intelligent algorithm by integrating bifuzzy simulation and genetic algorithm is designed. Finally, one numerical experiment is provided to illustrate effectiveness of the hybrid intelligent algorithm.
\end{abstract}

Keywords: Bifuzzy variable, Portfolio selection, Expected value operator, Risk curve, Hybrid intelligent algorithm

\section{Introduction}

The theory of portfolio selection was initially provided by Markowitz (1952, p.77) and has been greatly developed since then. It is concerned with selecting a combination of securities among portfolios containing large number of securities to reach the goal of obtaining satisfactory investment return. In his path-break work, Markowitz proposed a principle that when making investment decision, an investor should always strike a balance between maximizing the return and minimizing the risk, i.e., the investor maximize return for a given level of risk, or one should minimize risk for a predetermined return level. More importantly, Morkowitz initially quantified investment return as the expected value of returns of securities, and risk as variance from the expected value. After Maokowitz's work, scholars have been showing great enthusiasm in portfolio management, trying different mathematical approaches to develop the theory of portfolio selection. Traditionally, returns of individual security were assumed to be stochastic variables, and many researchers were focused on extending Markowitz's mean-variance models and on developing new mathematical approaches to solve the problems of computation. Zhou (2003, p.357) proposed concept of bifuzzy variable and the framework of bifuzzy system theory. In fact, investors may come across bifuzzy returns in portfolio selection situations. For example, security returns can be regarded as fuzzy variables in which still contain fuzzy parameter, to deal with this type of uncertainty, we propose the security returns could be regarded as bifuzzy variables. As a general mathematical description for this kind of fuzzy phenomenon with incomplete information, bifuzzy variable is defined as a mapping from a possibility space to a collection of fuzzy variables.

In general, there are three types of risk definitions in portfolio selection problems. Variance is the earliest and most commonly accepted definition of risk for portfolio selection initially proposed by Markowitz (1952, p.77). A variety of extensions to Markowitz's mean-variance models has been proposed. Semivariance is the second type of risk definitions, and was also proposed by Markowitz (1959). Semivariance is an improvement of variance because semivariance only measures portfolio return below the expected value, many models have been built to minimize semivariance in different cases. The third popular definition of risk is a probability of a bad outcome initially by Roy (1952 p.431). Much research has been undertaken to find ways of minimizing the probability of the bad outcome. Recently, Huang (2007 p.5404) proposed another new definition of risk for portfolio selection in fuzzy and random fuzzy environments. The detailed exposition on the definition of risk had been recorded in the literature ( Huang, 2007 p.5404), the interested readers may consult it. We can regard it as the fourth type of risk. Her work has enriched the risk theory for portfolio selection. We try to do something for portfolio selection in bifuzzy environments, and give a new risk definition in bifuzzy environments and a model for portfolio selection according to the proposed risk.

The rest of this paper is arranged as follows. After reviewing some necessary knowledge about bifuzzy variable in section 2 , in section 3, one type of risk for portfolio selection model under bifuzzy environment is proposed. In section 4, we give a model for portfolio selection from the point of the new definition of risk. To provide a general method for solving the new models, in section 5, a hybrid intelligent algorithm integrating genetic algorithm and bifuzzy simulation is designed. To better illustrate the modeling idea and demonstrate the effectiveness of the proposed algorithm, one numerical example is provided in section 6 . 


\section{Preliminaries}

Bifuzzy variable theory was introduced by Liu (2002, p.43) and developed by Zhou (2003, p.357). To better understand the expected value operator and the chance measure of bifuzzy variable, let us briefly review some necessary knowledge about bifuzzy variable.

Definition 1 A bifuzzy variable $\xi$ is a mapping from a possibility space $(\Theta, P(\Theta)$, Pos) to a collection of fuzzy variables.

Example 1 Let $\xi$ be a triangle fuzzy variable $(\rho-1, \rho, \rho+2)$, where $\rho$ is still a fuzzy variable with membership function $\mu_{\rho}(x)=[1-|x-1|] \vee 0$. Then $\xi$ is a bifuzzy variable.

The following are the definitions of the expected value operator and the primitive chance of bifuzzy event.

Definition 2 Let $\xi$ be a bifuzzy variable defined on the possibility space $(\Theta, P(\Theta)$, Pos). Then the expected value of bifuzzy variable $\xi$ is defined as

$$
E[\xi]=\int_{0}^{\infty} \operatorname{Cr}\{\theta \in \Theta \mid E[\xi(\theta)] \geq t\} d t-\int_{-\infty}^{0} \operatorname{Cr}\{\theta \in \Theta \mid E[\xi(\theta)] \leq t\} d t
$$

Provided that at least one of the above two integrals is finite.

Here, $\mathrm{Cr}$ denotes the credibility degree of fuzzy event and $E[\xi(\theta)]$ represents the expected value of fuzzy variable $\xi(\theta)$.

Definition 3 Let $\xi=\left(\xi_{1}, \xi_{2}, \cdots, \xi_{n}\right)$ be an n-dimensional bifuzzy vector defined on $\left(\Theta, P(\Theta)\right.$, Pos), and $B$ be a set of $R^{n}$. Then the primitive chance of bifuzzy event $\xi \in B$ is a function from $(0,1]$ to $[0,1]$, defined as

$$
\operatorname{Ch}\{\xi \in B\}(\delta)=\sup \{\beta \mid \operatorname{Cr}\{\theta \in \Theta \mid \operatorname{Cr}\{\xi(\theta) \in B\} \geq \beta\} \geq \delta\}
$$

Theorem 1 Let $\xi=\left(\xi_{1}, \xi_{2}, \cdots, \xi_{n}\right)$ be a bifuzzy vector on $\left(\Theta, P(\Theta)\right.$, Pos), and $f: R^{n} \rightarrow R^{m}$ a function. Then the chance $C h f(\xi) \leq 0$ is a decreasing and left-continuous function of $\alpha$.

Theorem 2 Let $\xi$ be a bifuzzy variable and $\alpha$ a given number in $(0,1]$, then the chance distribution $\operatorname{Ch}\{\xi \geq x\}(\alpha)$ is a decreasing and left-continuous function of $x$.

\section{New definition of risk}

In reality, some investors are only sensitive to one preset bad case. They regard as safe those securities whose chance of this bad case occurring is lower than the investors' tolerance level. Other investors consider all the possible unfavorable cases, and only those securities whose chance of every unfavorable case occurring is lower than the investors' tolerance level are regarded as safe. We will define the risk from this perspective.

Definition 4 Let $\xi$ be a bifuzzy variable on the possibility space $(\Theta, P(\Theta)$, Pos), and $\delta$ the preset confidence level and $b$ the target return. Then the curve

$$
f(\delta, r)=C h\{b-\xi \geq r\}(\delta), \quad \forall r \in R
$$

is called the risk curve of an investment in the portfolio, and $r$ the loss severity indicator.

The greater the indicator $r$, the more severe the loss $b-\xi$. the risk curve $f(\delta, r)$ gives the chance of the occurrence of all events when the bifuzzy return $\xi$ is $r$ less than the target return $b$.

From theorem 1 and theorem 2 we can derive that the risk curve $f(\delta, r)$ is a decreasing function with respect to $\delta$ and $r$, that is, the greater the $\delta$, the smaller the $f(\delta, r)$ value, the greater the $r$, the smaller the $f(\delta, r)$.

To determine whether a portfolio selection is risky, an investor must first decide what is his or her maximum tolerance level of each bad event occurring. Usually, the worse the event, the lower the tolerance level. Then for every loss severity indicator $r_{0} \in R$, the investor always can give a confidence level corresponding $\alpha\left(r_{0}\right)$, thus the confidence level $\alpha(r)$ is a function of the loss severity indicator $r$, the function $\alpha(r)$ is called the confidence curve.

Let $\xi$ be a bifuzzy return of a portfolio $A$, and $\alpha(r)$ the confidence curve. We can say that $A$ is safe if $f(\delta, r)=C h\{b-\xi \geq$ $r\}(\delta) \leq \alpha(r)$ for every $r \in R$, where $b$ is the target return and $\delta$ the preset credibility level. The number $r$ denotes all possible loss severity indicator. If the investor is only concern with one special loss severity indicator $r_{0}$, then the risk becomes the chance $C h\left\{b-\xi \geq r_{0}\right\}(\delta)$, which is exactly the ordinary chance measure of bifuzzy variable.

\section{Bifuzzy portfolio selection}

Let us select a portfolio according to the definition of risk in the preceding content. Let $x_{i}$ denotes the investment proportions in security $i, \xi_{i}$ the bifuzzy return for the ith security, $i=1,2, \cdots, n$, respectively. Let $r$ denote the loss severity indicator, and $\alpha(r)$ the confidence curve preset by the investor. To obtain the maximum investment return and avoid risk, the investor should select an optimal combination of securities from the portfolio safe point. We use the expected value of the securities to express the investment return. Thus we should set a goal of maximizing the expected return of a portfolio, 
and require that the risk curve $f(\delta, r)$ is not larger than the confidence curve $\alpha(r)$. Let $b$ be the target return and $\delta$ the preset credibility level. Then the model is formulated as follows:

$$
\begin{gathered}
\max _{x} E\left[\sum_{i=1}^{n} x_{i} \xi_{i}\right] \\
\text { subject to } \\
C h\left\{b-\sum_{i=1}^{n} x_{i} \xi_{i} \geq r\right\}(\delta) \leq \alpha(r), \forall r \in R \\
\sum_{i=1}^{n} x_{i}=1 \quad x_{i} \geq 0, i=1,2, \cdots, n
\end{gathered}
$$

when the bifuzzy returns degenerate to fuzzy, the chance constraint becomes $\operatorname{Cr}\left\{b-\sum_{i=1}^{m} \xi_{i} x_{i} \geq r\right\} \leq \alpha(r), \forall r \in R$, for any given $\delta \in(0,1]$. Thus the model is the following

$$
\begin{gathered}
\max _{x} E\left[\sum_{i=1}^{n} x_{i} \xi_{i}\right] \\
\text { subject to } \\
C r\left\{b-\sum_{i=1}^{n} x_{i} \xi_{i} \geq r\right\} \leq \alpha(r), \forall r \in R \\
\sum_{i=1}^{n} x_{i}=1 \quad x_{i} \geq 0, i-1,2, \cdots, n
\end{gathered}
$$

Furthermore, if the investor only concerns one preset loss severity level $r_{0}$, then the model (2) can be converted into the formulation:

$$
\begin{gathered}
\max _{x} E\left[\sum_{i=1}^{n} x_{i} \xi_{i}\right] \\
\text { subject to } \\
C r\left\{b-\sum_{i=1}^{n} x_{i} \xi_{i} \geq r_{0}\right\} \leq \alpha\left(r_{0}\right) \\
\sum_{i=1}^{n} x_{i}=1 \quad x_{i} \geq 0, i=1,2, \cdots, n
\end{gathered}
$$

\section{Hybrid intelligent algorithm}

Since the two-fold uncertainty of bifuzzy variable, it is difficult to analytically solve the models (1), (2) and (3). To provide a general solution to the models, we design a hybrid intelligent algorithm integrating genetic algorithm (GA) and bifuzzy simulation. Roughly speaking, in the proposed hybrid intelligent algorithm, the technique of bifuzzy simulation is applied to compute the expected value and the chance measure, then bifuzzy simulation and GA are integrated for solving the bifuzzy models.

\subsection{Bifuzzy simulation}

In this section, we first discuss the calculation of the expected value and the chance measure of bifuzzy variables. Let $\xi_{i}$ be bifuzzy variables and $x_{i}$ decision variables, $i=1,2, \cdots, n$, respectively. Write $f(x, \xi)=\sum_{i=1}^{n} x_{i} \xi_{i}$,

where $x=\left(x_{1}, x_{2}, \cdots, x_{n}\right), \xi=\left(\xi_{1}, \xi_{2}, \cdots, \xi_{n}\right)$. Let $b$ be the target return and $\delta$ the preset credibility level. The number $r$ denotes all possible loss severity indicator. In order to solve the proposed models, we must handle the following two types of uncertain function:

$$
\begin{gathered}
U_{1}: x \rightarrow E\{f(x, \xi)\} \\
U_{2}: x \rightarrow C h\{b-f(x, \xi) \geq r\}(\delta) \leq \alpha(r), \forall r \in R
\end{gathered}
$$

A bifuzzy simulation will be introduced to compute the expected value $E[f(x, \xi)]$. We randomly sample $\theta_{k}$ from $\Theta$ such that $\operatorname{Pos}\left\{\theta_{k}\right\} \geq \varepsilon$, and Write $v_{k}=\operatorname{Pos}\left\{\theta_{k}\right\}, k=1,2, \cdots, N$, respectively, where $\varepsilon$ is a sufficiently small number. Then for any number $c \geq 0$, the credibility $\operatorname{Cr}\left\{\theta \in \Theta \mid E\left[f\left(x, \xi\left(\theta_{k}\right)\right)\right] \geq c\right\}$ can be estimated by 


$$
\left[\left(\max _{1 \leq k \leq N}\left\{v_{k} \mid E\left[f\left(x, \xi\left(\theta_{k}\right)\right)\right] \geq c\right\}+\max _{1 \leq k \leq N}\left\{1-v_{k} \mid E\left[f\left(x, \xi\left(\theta_{k}\right)\right)\right]<c\right\}\right] / 2\right.
$$

and for any number $c<0$, the credibility $\operatorname{Cr}\{\theta \in \Theta \mid E[f(x, \xi(\theta))] \leq c\}$ can be estimated by

$$
\left[\left(\max _{1 \leq k \leq N}\left\{v_{k} \mid E\left[f\left(x, \xi\left(\theta_{k}\right)\right)\right] \leq c\right\}\right)+\max _{1 \leq k \leq N}\left\{1-v_{k} \mid E\left[f\left(x, \xi\left(\theta_{k}\right)\right)\right]>c\right\}\right] / 2
$$

Provided that $N$ is sufficiently large, and $E\left[f\left(x, \xi\left(\theta_{k}\right)\right)\right], k=1,2, \cdots, N$, may be estimated by the fuzzy simulation. We describe the process as follows.

Algorithm 1 (bifuzzy simulation for $U_{1}(x)$ )

Step 1. Set $E=0$

Step 2. Randomly sample $\theta_{k}$ from $\Theta$ such that $\operatorname{Pos}\left\{\theta_{k}\right\} \geq \varepsilon, k=1,2, \cdots, N$, where $\varepsilon$ is a sufficiently small number.

Step 3. Compute $E\left[f\left(x, \xi\left(\theta_{k}\right)\right)\right]$ by fuzzy simulation for $k=1,2, \cdots, N$, respectively.

Step 4. Let $\alpha=\min _{1 \leq K \leq N} E\left[f\left(x, \xi\left(\theta_{k}\right)\right)\right]$ and $b=\max _{1 \leq k \leq N} E\left[f\left(x, \xi\left(\theta_{k}\right)\right)\right]$.

Step 5. Randomly generate $c$ from $[a, b]$.

Step 6. If $c \geq 0$, then $E \leftarrow E+\operatorname{Cr}\left\{\theta \in \Theta \mid E\left[f\left(x, \xi\left(\theta_{k}\right)\right)\right] \geq c\right\}$, where fuzzy simulation is used to calculate $\operatorname{Cr}\{\theta \in$ $\left.\Theta \mid E\left[f\left(x, \xi\left(\theta_{k}\right)\right)\right] \geq c\right\}$. If $c<0$, then $E \leftarrow E-\operatorname{Cr}\left\{\theta \in \Theta \mid E\left[f\left(x, \xi\left(\theta_{k}\right)\right)\right] \leq c\right\}$, where fuzzy simulation is also used to calculate $\operatorname{Cr}\left\{\theta \in \Theta \mid E\left[f\left(x, \xi\left(\theta_{k}\right)\right)\right] \leq c\right\}$.

Step 7. Repeat the fifth to the seventh steps for $N$ times.

Step 8. $E[f(x, \xi)]=a \vee 0+b \wedge 0+E(b-a) / N$.

For any given confidence level $\delta$ and given $b, r x$, we design a bifuzzy simulation to compute the $\alpha-\operatorname{chance} L=C h\{b-$ $f(x, \xi) \geq r\}(\delta)$. Equivalently, we should estimate $L=\sup \{\beta \mid \operatorname{Cr}\{\theta \in \Theta \mid \operatorname{Cr}\{b-f(x, \xi(\theta)) \geq r\} \geq \beta\} \geq \delta\}$. We randomly generate $\theta_{k}$ from $\Theta$ such that $\operatorname{Pos}\left\{\theta_{k}\right\} \geq \varepsilon$, and write $v_{k}=\operatorname{Pos}\left\{\theta_{k}\right\}, k=1,2, \cdots, N$, respectively, where $\varepsilon$ is a sufficiently small number. For any number $\theta_{k}$, by using fuzzy simulation, we can estimate the credibility $h\left(\theta_{k}\right)=\operatorname{Cr}\left\{f\left(x, \xi\left(\theta_{k}\right)\right) \geq r\right\}$. For any number $c$, we have

$$
L(c)=\left[\left(\max _{1 \leq k \leq N}\left\{v_{k}\left(\theta_{k}\right) \geq c\right\}+\min _{1 \leq k \leq N}\left\{1-v_{k} \mid h\left(\theta_{k}\right)<c\right\}\right)\right] / 2
$$

It follows from monotony that we may use bisection search to find the maximal value $c$ such that $L(c) \geq \alpha$. This value is an estimate of $L$. We summarize this process as follows.

Algorithm 2 (bifuzzy simulation for $U_{2}(x)$ )

Step 1. Set $l=1$.

Step 2. Randomly generate a real number $r$ according to the confidence curve $\alpha(r)$ given by the investor.

Step 3. Generate $\theta_{k}$ from $\Theta$ such that $\operatorname{Pos}\left\{\theta_{k}\right\} \geq \varepsilon$, and write $v_{k}=\operatorname{Pos}\left\{\theta_{k}\right\}, k=1,2, \cdots, N$, respectively, where $\varepsilon$ is a sufficiently small number.

Step 4. Compute $h\left(\theta_{k}\right)=C r\left\{f\left(x, \xi\left(\theta_{k}\right)\right) \geq R\right\}$ by fuzzy simulation, $k=1,2, \cdots, N$, respectively.

Step 5. Find the maximal value $c$ such that $L(c) \geq \alpha$ holds, where $L(c)$ is defined by (4).

Step 6. Return $c$.

Step 7. If $c$ is no larger than $\alpha(r)$, then $l=l * 1$, else $l=l * 0$.

Step 8. repeat the second to the fifth steps for a given number times.

Step 9. If $l=1$, then return YES, else return NO.

Remark: here YES means that the investment proportion $x$ is feasible; NO means that $x$ is infeasible.

\subsection{Genetic algorithm}

Representation structure: A solution $x=\left(x_{1}, x_{2}, \cdots, x_{n}\right)$ is represented by the chromosome $V=\left(v_{1}, v_{2}, \cdots, v_{n}\right)$, where the genes $v_{1}, v_{2}, \cdots, v_{n}$ are randomly generated in the interval [0,1], and the relation between $x$ and $V$ are formulated as follows: $x_{i}=v_{i} /\left(v_{1}+v_{2}+\cdots+v_{n}\right), i=1,2, \cdots, n$, which ensures that $x_{1}+x_{2}+\cdots+x_{n}=1, x_{i} \geq 0, i=1,2, \cdots, n$ always holds.

Initialization process: pop_size number of chromosomes are initialized randomly by generating points $\left(v_{1}, v_{2}, \cdots, v_{n}\right)$ from the hypercube $[0,1]^{n}$ pop-size times. Since the constraint required that $x=\left(x_{1}, x_{2}, \cdots, x_{n}\right)$ satisfy $x_{1}+x_{2}+\cdots+x_{n}=$ 1 , based on the relation between $x$ and $V$, the feasibility of the randomly generated chromosomes is obvious. 
Evaluation function: Evaluation function, denoted by $E v a(V)$, is to assign a probability of reproduction to each chromosome $V$ so that its likelihood of being selected is proportional to its fitness relative to the other chromosomes in the population. That is, the chromosomes with higher fitness will have more chance to produce offspring by using roulette wheel selection. One well-known evaluation function is based on allocation of reproductive trial according to rank rather than actual objective values. We can rearrange the pop-size chromosomes according to their objective values to make better chromosome take smaller ordinal number. That is, after rearrange, among pop-size chromosomes $V_{1}, V_{2}, \cdots, V_{\text {pop_size }}, V_{1}$, is the best chromosome, and $V_{\text {pop_size }}$ the worst one, then a parameter $\alpha \in(0,1)$ in the genetic system is given. We can define the rank-based evaluation function as follows: $E v a\left(V_{i}\right)=a(1-a)^{i}, i=1,2, \cdots, p o p \_s i z e$. Note that $i=1$ means the best individual, $i=$ pop_size the worst one.

Selection process: Firstly, calculate the cumulative probability $q_{i}$ for each chromosome $V_{i}$,

$$
q_{0}=0, q_{i}=\sum_{j=i}^{i} \operatorname{Eva}\left(V_{j}\right), i=1,2, \cdots, \text { pop_size }
$$

Secondly, generate a random number $r$ in $\left(0, q_{\text {pop_size }}\right]$, and select the chromosome $V_{i}$ if $r$ satisfies $q_{i-1}<r \leq q_{i}$. Repeat the second and third steps pop_size times and obtain pop_size copies of chromosome.

Crossover operation: A parameter $p_{c}$ of a genetic system as the probability of crossover is defined first. The parents for crossover operation are selected by doing the following process repeatedly from $i=1$ to pop $_{s} i z e$ : Generating a random number $r$ from the interval [0,1], the chromosome $V_{i}$ is selected as a parent if $r<p_{c}$, the selected parents are denoted by $V_{1}^{\prime}, V_{2}^{\prime}, V_{3}^{\prime}, \cdots$ and divided into the pairs: $\left(V_{1}^{\prime}, V_{2}^{\prime}\right),\left(V_{3}^{\prime}, V_{4}^{\prime}\right),\left(V_{5}^{\prime}, V_{6}^{\prime}\right), \cdots$. The crossover operation on each pair is illustrated by $\left(V_{1}^{\prime}, V_{2}^{\prime}\right)$. At first, we generate a random number $c$ from the open interval $(0,1)$, then the operator on $V_{1}^{\prime}$ and $V_{2}^{\prime}$ will product two children $X$ and $Y$ as follows: $X=c V_{1}^{\prime}+(1-c) V_{2}^{\prime}, Y=(1-c) V_{1}^{\prime}+c V_{2}^{\prime}$.

If both children are feasible, then we replace the parents with them. If not, we keep the feasible one if it exists, and then redo the crossover operator by regenerating a random number $c$ until two feasible children are obtained or a given number of cycles is finished. In this case, we only replace the parents with the feasible children .

Mutation operation: A parameter $p_{m}$ of a genetic system as the probability of mutation is defined first. This probability gives us the expected number of $p_{m} \cdot$ pop_size of chromosomes undergoing the mutation operations. We repeat the following steps from $i=1$ to pop_size: Generating a random number $r$ from the interval $[0,1]$, the chromosome $V_{i}$ is selected as a parent if $r<p_{m}$. For each selected parents $V_{i}$, we mutate it in the following way. Let $M$ be an appropriate large positive number. We choose a mutation direction $d$ in $R^{n}$ randomly. If $V+M \cdot d$ is not feasible, then we set $M$ as a random number between 0 and $M$ until it is feasible. If the above process cannot find a feasible solution in a predetermined number of iterations, then we set $M=0$. Anyway, we replace the parent $V_{i}$ with its feasible child $V+M \cdot d$.

The following is the hybrid intelligent algorithm integrating bifuzzy simulation and genetic algorithm.

Algorithm 3 (hybrid intelligent algorithm)

Step 1. Initialize pop_size chromosomes.

Step 2. Calculate the objective values for all chromosomes by bifuzzy simulation.

Step 3. Given the rank order of the chromosomes according to the objective values, and the values of the rank-based evaluation function of the chromosomes.

Step 4 . Mpute the fitness of each chromosome according to the rank-based evaluation function.

Step 5. lect the chromosomes by spinning the roulette wheel.

Step 6. Update the chromosomes by crossover and mutation operations.

Step 7. Repeat the second step to the sixth step for a given number of cycles.

Step 8. Take the best chromosome as the solution of portfolio selection.

\section{Numerical example}

To illustrate the modeling idea and to test the effectiveness of the designed hybrid intelligent algorithm, let us consider one numerical example. The example is performed on a personal computer by using $C++$ programming language. The parameters in the HIA are set as follows: the probability of crossover $p_{c}=0.3$, the probability of mutation $p_{m}=0.2$, the parameter $a=0.05$ in the rank-based evaluation function.

Example 2 Assume that there are 5 securities, the returns of securities are all bifuzzy variables.

$$
\max _{x} E\left[\sum_{i=1}^{5} x_{i} \xi_{i}\right]
$$




$$
\begin{gathered}
\text { subject to } \\
C h\left\{b-\sum_{i=1}^{5} x_{i} \xi_{i} \geq r\right\}(\delta) \leq \alpha(r), \forall r \in R \\
\sum_{i=1}^{5} x_{i}=1, \quad x_{i} \geq 0, i=1,2, \cdots, 5
\end{gathered}
$$

where

$\xi_{1}=\left(\rho_{1}-1, \rho_{1}, \rho_{1}+1\right)$ with $\rho_{1}=(0,1,2), \xi_{2}=\left(\rho_{2}-1, \rho_{2}, \rho_{2}+1\right)$ with $\rho_{2}=(1,2,3)$

$\xi_{3}=\left(\rho_{3}-1, \rho_{3}, \rho_{3}+1\right)$ with $\rho_{3}=(2,3,4), \xi_{4}=\left(\rho_{4}-1, \rho_{4}, \rho_{4}+1\right)$ with $\rho_{4}=(3,4,5)$

$\xi_{5}=\left(\rho_{5}-1, \rho_{5}, \rho_{5}+1\right)$ with $\rho_{5}=(4,5,6)$

Suppose that the investor has given the confidence curve and other parameters: $\alpha(r)=\frac{1}{r^{2}+1}, r \geq 0 . b=0.9, \delta=0.9$.

A run of the hybrid intelligent algorithm with 3000 generations shows that among 5 securities, in order to gain maximum expected value of the total securities return the investor should assign his money according to the optimal solution:

$$
x_{i}^{*}=0, x_{2}^{*}=0.1325, x_{3}^{*}=0.2536, x_{4}^{*}=0.2792, x_{5}^{*}=0.3347
$$

\section{References}

A. Charnes \& W. W. Cooper. (1965). Chance-constrained programming. Management Science, 6, 73-79.

A. D. Roy. (1952). Safety first and the holding of assets. Econometrics, 20, 431-449.

H. Markowitz. (1952). Portfolio selection. Journal of Finance, 7, 77-91.

H. Markowitz. (1959). Portfolio selection: Efficient diversification of investment. New York :Wiley.

Huang, Xiaoxia. (2006). Fuzzy chance-constrained portfolio selection. Applied mathematics and computation, 177, 500-507.

Huang, Xiaoxia. (2007). A new perspective for optimal portfolio selection with random fuzzy returns. Information science, 177, 5404-5414.

Liu , B. Uncertainty theory, 3rd edition. [Online] Available: http://orsc.edu.cn/lliu/ut.pdf.

Liu. B. (2002). Toward fuzzy optimization without mathematical ambiguity. Fuzzy optimization and decision making, 1 , 43-63.

Zhou, Jian. (2003). Analysis and algorithms of bifuzzy systems. International Journal of uncertainty, fuzziness and knowledge-based systems, 12, 357-376. 